\title{
Solid Inclusion Complexes of Vanillin with Cyclodextrins: Their Formation, Characterization, and High-Temperature Stability
}

\author{
Fatma Kayaci and Tamer Uyar* \\ UNAM-Institute of Materials Science and Nanotechnology, Bilkent University, Ankara 06800, Turkey
}

\begin{abstract}
This study reports the formation of solid vanillin/cyclodextrin inclusion complexes (vanillin/CD ICs) with the aim to enhance the thermal stability and sustained release of vanillin by inclusion complexation. The solid vanillin/CD ICs with three types of $\mathrm{CDs}(\alpha-\mathrm{CD}, \beta-\mathrm{CD}$, and $\gamma$-CD) were prepared using the freeze-drying method; in addition, a coprecipitation method was also used in the case of $\gamma$-CD. The presence of vanillin in CD ICs was confirmed by FTIR and ${ }^{1} \mathrm{H}$ NMR studies. Moreover, ${ }^{1} \mathrm{H}$ NMR study elucidated that the complexation stoichiometry for both vanillin $/ \beta$-CD IC and vanillin $/ \gamma$-CD IC was a 1:1 molar ratio, whereas it was $0.625: 1$ for vanillin $/ \alpha$-CD IC. XRD studies have shown channel-type arrangement for CD molecules, and no diffraction peak for free vanillin was observed for vanillin $/ \beta$-CD IC and vanillin $/ \gamma$-CD IC, indicating that complete inclusion complexation was successfully achieved for these CD ICs. In the case of vanillin $/ \alpha$-CD IC, the sample was mostly amorphous and some uncomplexed vanillin was present, suggesting that $\alpha$-CD was not very effective for complexation with vanillin compared to $\beta$-CD and $\gamma$-CD. Furthermore, DSC studies for vanillin $/ \beta$-CD IC and vanillin $/ \gamma$-CD IC have shown no melting point for vanillin, elucidating the true complex formation, whereas a melting point for vanillin was recorded for vanillin $/ \alpha$-CD IC, confirming the presence of some uncomplexed vanillin in this sample. TGA thermograms indicated that thermal evaporation/degradation of vanillin occurred over a much higher temperature range $\left(150-300^{\circ} \mathrm{C}\right)$ for vanillin/CD ICs samples when compared to pure vanillin $\left(80-200^{\circ} \mathrm{C}\right)$ or vanillin/CD physical mixtures, signifying that the thermal stability of vanillin was increased due to the inclusion complexation with CDs. Moreover, headspace GC-MS analyses indicated that the release of vanillin was sustained at higher temperatures in the case of vanillin/CD ICs due to the inclusion complexation when compared to vanillin/CD physical mixtures. The amount of vanillin released with increasing temperature was lowest for vanillin $/ \gamma$-CD IC and highest for vanillin $/ \alpha$-CD IC, suggesting that the strength of interaction between vanillin and the $\mathrm{CD}$ cavity was in the order $\gamma-\mathrm{CD}>\beta-\mathrm{CD}>\alpha-\mathrm{CD}$ for solid vanillin/CD ICs.
\end{abstract}

KEYWORDS: cyclodextrin, vanillin, inclusion complex, thermal stability, sustained release

\section{INTRODUCTION}

Cyclodextrin inclusion complexation is widely used in the food, cosmetics, and textile industries to increase thermal stability and to control/sustain the release of volatile flavors and fragrances or other functional additives. ${ }^{1-9}$ Cyclodextrins (CDs) are cyclic oligosaccharides that are produced by the enzymatic degradation of starch. ${ }^{1}$ The three most common CDs have 6, 7, and 8 glucopyranose units in the cyclic and are named $\alpha$-CD, $\beta$-CD, and $\gamma-\mathrm{CD}$, respectively. Whereas the depth of the cavity for these $\mathrm{CDs}$ is $\sim 8 \AA$, the sizes of the cavity are different for $\alpha$-CD, $\beta$-CD, and $\gamma-\mathrm{CD}$, being $\sim 6,8$, and $10 \AA$, respectively (Figure $1 \mathrm{~b}) .{ }^{1,2}$ CDs have a truncated cone-shaped molecular structure having a relatively hydrophobic inner cavity and hydrophilic outer surface because of their unique chemical structure. The hydrophobic cavities of CDs have a remarkable ability to form noncovalent host-guest inclusion complexes (ICs) with a variety of molecules. ${ }^{1-9}$ The formation and stability of the CD ICs depend on various factors including the size/shape fit, chemical surroundings, and binding forces (hydrophobic interactions, van der Waals attractions, hydrogen bonding, electrostatic interactions, etc.) between the host CD and guest molecules. ${ }^{10-12}$ The physical and chemical properties of the guest molecules are improved when complexed with CDs; therefore, CD ICs are quite applicable in the food, cosmetics, and textile industries because CDs enhance the stabilization and controlled/ sustained release of volatile or unstable flavors and fragrances. ${ }^{1-9}$
Vanillin (4-hydroxy-3-methoxybenzaldehyde) (Figure 1e), which is the major component of natural vanilla, is widely used as a flavoring additive in the food industry. ${ }^{13-15}$ Vanillin can also be used as a food preservative due to its antioxidant and antimicrobial properties. ${ }^{14,15}$ Moreover, vanillin is used as a fragrance constituent in the cosmetics and textile industries. ${ }^{15,16}$ However, a short shelf life is a significant problem for vanillin due to its thermal instability and volatile nature. Here, we studied the formation of vanillin/cyclodextrin inclusion complexes (vanillin/CD ICs) for the enhancement of the thermal stability and sustained release of vanillin by using three types of CDs, $\alpha-\mathrm{CD}, \beta-\mathrm{CD}$, and $\gamma-\mathrm{CD}$. Very few studies have been reported for the formation of vanillin/CD ICs by using $\beta$-CD, and these CD ICs were mostly studied in solutions. ${ }^{14,17-20}$ However, to date, the solid vanillin/ CD IC systems have not been studied in detail, and the inclusion complexation of vanillin with different types of CDs has not been evaluated in the solid state. In this study, we formed solid vanillin/CD ICs by using $\alpha-\mathrm{CD}, \beta-\mathrm{CD}$, and $\gamma-\mathrm{CD}$ by a freezedrying method and coprecipitation method (only for $\gamma$-CD), and we have carried out extensive characterization of these vanillin/CD ICs by using FTIR, ${ }^{1} \mathrm{H}$ NMR, XRD, DSC, TGA, and headspace

Received: July 22, 2011

Revised: September 17, 2011

Accepted: September 19, 2011

Published: September 20, 2011 


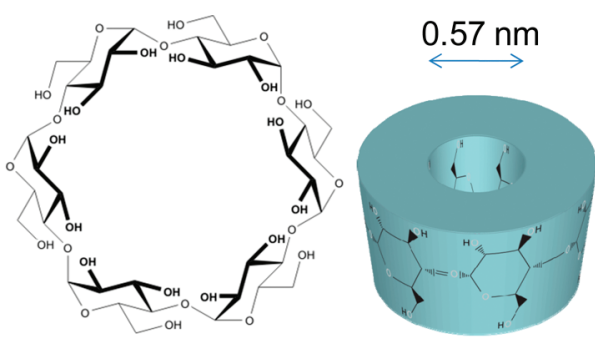

a.

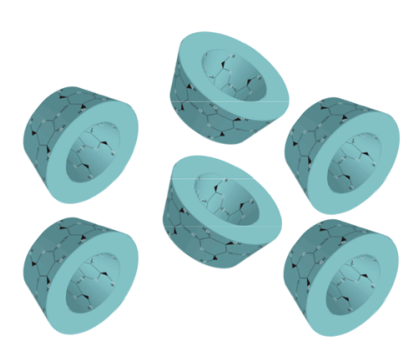

c.

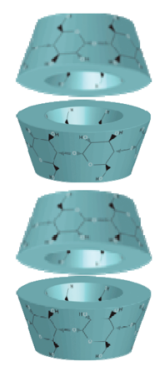

d.
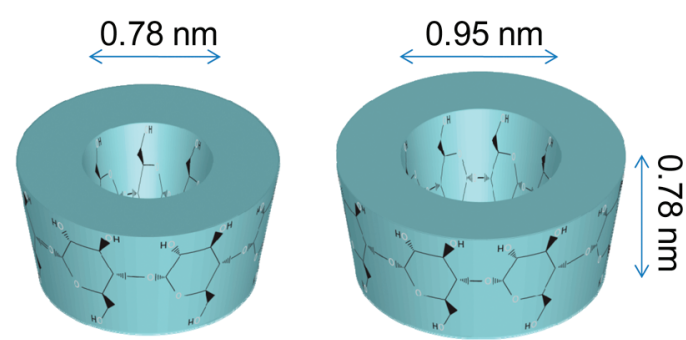

b.

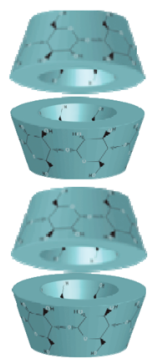

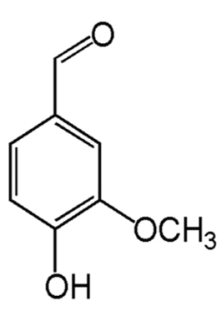

e.

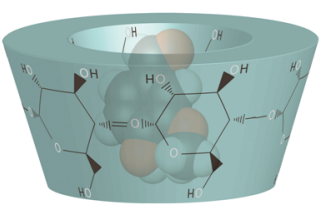

f.

Figure 1. (a) Chemical structure of $\alpha$-CD; (b) approximate dimensions of $\alpha-\mathrm{CD}, \beta-\mathrm{CD}$, and $\gamma$-CD; schematic representation of packing structures of (c) cage-type and (d) channel-type CD crystals; (e) chemical structure of vanillin, and (f) schematic representation of vanillin/CD IC.

GC-MS techniques. The physical mixtures of vanillin/CDs and pure vanillin were also analyzed for comparison.

\section{MATERIALS AND METHODS}

Materials. Vanillin (4-hydroxy-3-methoxybenzaldehyde, $\mathrm{C}_{8} \mathrm{H}_{8} \mathrm{O}_{3}$; molecular weight, 152.15 ; melting point, $81-83{ }^{\circ} \mathrm{C}$; $99 \%$ purity) was obtained from Sigma-Aldrich. Cyclodextrins $(\alpha-\mathrm{CD}, \beta$-CD, and $\gamma$-CD) were purchased from Wacker Chemie AG (Germany). All materials were used without any purification. Water used for the preparation of the complexes was from a Millipore Milli-Q ultrapure water system.

Preparation of Vanillin/CD ICs. The inclusion complexes of vanillin with $\alpha$-CD, $\beta$-CD, and $\gamma-\mathrm{CD}$ were prepared by adding an equimolar amount of vanillin to an aqueous solution of $\alpha-\mathrm{CD}, \beta-\mathrm{CD}$, and $\gamma$-CD. The amount of the $\mathrm{CD}$ used was determined according to the solubility of CDs in water at $25^{\circ} \mathrm{C}$, that is, $14.5,1.85$, and $23.2 \mathrm{~g} / 100 \mathrm{~mL}$ for $\alpha$-CD, $\beta$-CD, and $\gamma-\mathrm{CD}$, respectively. ${ }^{1,2}$ First, $0.25 \mathrm{~g}$ of $\alpha$-CD dissolved in $1.725 \mathrm{~mL}$ of water and, then, $0.039 \mathrm{~g}$ of vanillin were added into the aqueous $\alpha$-CD solution, and the vanillin/ $\alpha-\mathrm{CD}$ solution was mixed overnight at room temperature. The vanillin $/ \alpha-\mathrm{CD}$ solution became slightly turbid, but precipitation did not occur. For vanillin/ $\beta$-CD IC, $0.25 \mathrm{~g}$ of $\beta$-CD was dissolved in $14 \mathrm{~mL}$ of water, and then $0.034 \mathrm{~g}$ of vanillin was added into this clear $\beta$-CD aqueous solution. The vanillin/ $\beta$-CD solution became clear after mixing overnight at room temperature, and no precipitation was observed. In the case of vanillin/ $\gamma$-CD IC, $0.25 \mathrm{~g}$ of $\gamma$-CD was dissolved in $1.08 \mathrm{~mL}$ of water and $0.029 \mathrm{~g}$ of vanillin was added into this solution. The vanillin $/ \gamma$-CD solution first became clear and then highly turbid and precipitation took place after mixing overnight at room temperature. Thereafter, all three vanillin/CD solutions were frozen at $-80{ }^{\circ} \mathrm{C}$ and then lyophilized in a freeze-dryer (Labconco) to obtain solid vanillin/CD ICs. In addition, the inclusion complex of vanillin with $\gamma$-CD was also prepared by a coprecipitation method. Similarly, $0.029 \mathrm{~g}$ of vanillin was added to an aqueous solution of $\gamma$-CD ( $0.25 \mathrm{~g}$ of $\gamma$-CD dissolved in $1.08 \mathrm{~mL}$ of water $)$ and stirred overnight. The resulting suspension was filtered through a borosilicate filter (por 2), and then the filtrate was washed with water several times to remove uncomplexed vanillin and $\gamma-\mathrm{CD}$ and then dried overnight under a hood. After drying, the vanillin $/ \gamma$-CD IC solid sample was crushed in a mortar to obtain a fine powder. For comparison, physical mixtures of vanillin/CDs having a 1:1 molar ratio were prepared in the solid state by admixing vanillin and $\mathrm{CDs}$ and ground with a pestle in an agar mortar.

Characterization and Measurements. A Fourier transform infrared (FTIR) spectrometer (VERTEX70, Bruker) was used to obtain the infrared spectra of the samples. The FTIR measurements were performed by blending the samples with potassium bromide $(\mathrm{KBr})$, and pellets were formed under high pressure. The spectra were recorded between 4000 and $400 \mathrm{~cm}^{-1}$ with a resolution of $4 \mathrm{~cm}^{-1}$ at total scans of 64. The proton nuclear magnetic resonance $\left({ }^{1} \mathrm{H}\right.$ NMR, Bruker DPX-400) spectra were recorded at $400 \mathrm{MHz}$ at $25^{\circ} \mathrm{C}$. About $20 \mathrm{~g} / \mathrm{L}$ vanillin/CD ICs was dissolved in $d_{6}$-DMSO to evaluate the stoichiometries of the complexes. Integration of the chemical shifts $(\delta)$ given in parts per million (ppm) of the samples was calculated by using NMR software. The X-ray diffraction (XRD, PANalyticalX'Pert Powder diffractometer) patterns for the samples were collected by using $\mathrm{Cu} \mathrm{K} \alpha$ radiation in a range of $2 \theta=5-30^{\circ}$. The thermal properties of the vanillin/CD ICs, vanillin/CD physical mixtures, and pure vanillin were investigated by using a differential scanning calorimeter (DSC, Q2000, TA Instruments) and a thermogravimetric analyzer (TGA, Q500, TA Instruments). For DSC analyses, the samples were initially equilibrated at $25^{\circ} \mathrm{C}$ and then heated to $250{ }^{\circ} \mathrm{C}$ at a $20^{\circ} \mathrm{C} / \mathrm{min}$ heating rate under $\mathrm{N}_{2}$ as a purge gas. TGA measurements were performed from room temperature to $500^{\circ} \mathrm{C}$ at a heating rate of $20^{\circ} \mathrm{C} / \mathrm{min}$ under $\mathrm{N}_{2}$ atmosphere.

Headspace gas chromatography-mass spectrometry (GC-MS) analyses were performed on an Agilent Technologies 7890A gas chromatograph coupled to an Agilent Technologies 5975C inert MSD with a triple-axis detector. The headspace GC-MS experiments were carried out by using a CTCPAL autosampler. Different amounts of CD ICs ( $0.0185 \mathrm{~g}$ vanillin $/ \alpha$-CD IC, $0.0211 \mathrm{~g}$ vanillin $/ \beta$-CD IC, and $0.238 \mathrm{~g}$ vanillin $/ \gamma$-CD IC) were placed separately in $20 \mathrm{~mL}$ headspace glass vials to have the same amount of vanillin $(0.0025 \mathrm{~g})$ in each vial. For comparison, the same amounts of vanillin/CD physical mixtures were placed separately in $20 \mathrm{~mL}$ headspace glass vials for the headspace GC-MS measurements. The tops of all vials were tightened to prevent the escape of the vanillin. Every sample was agitated at $250 \mathrm{rpm}$ at different incubation temperatures in the order $35,50,75$, and $100^{\circ} \mathrm{C}$ for $30 \mathrm{~min}$ by using an agitator and then analyzed by headspace GC-MS. One thousand 
a.

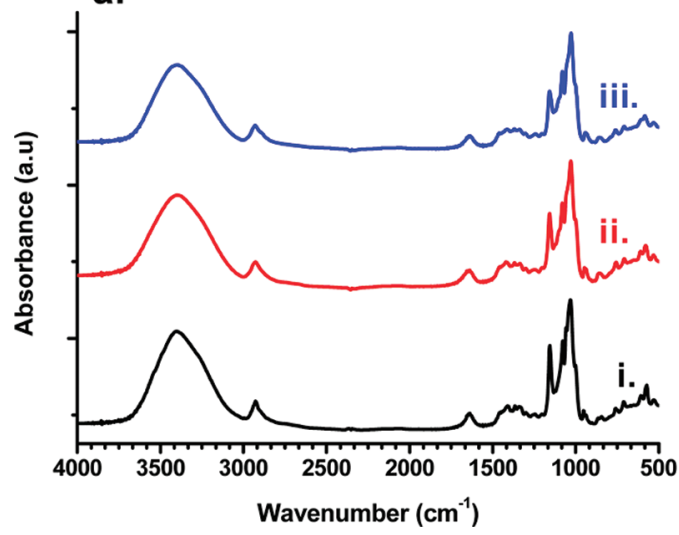

b.

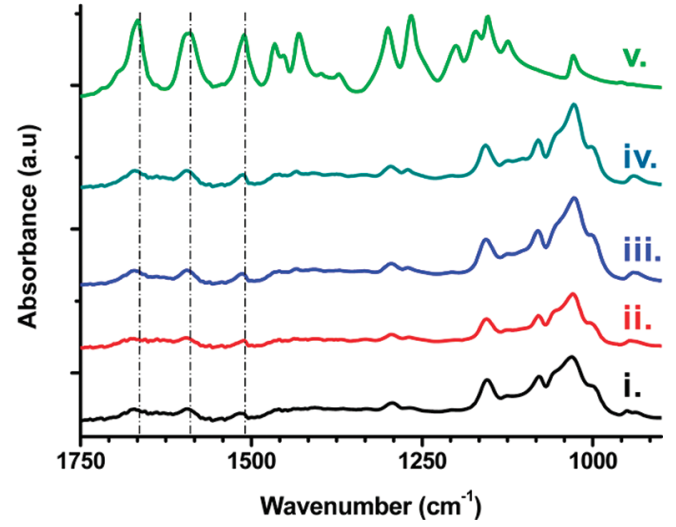

Figure 2. FTIR spectra of (a) as-received (i) $\alpha$-CD, (ii) $\beta$-CD, and (iii) $\gamma$-CD and (b) (i) vanillin $/ \alpha$-CD IC, (ii) vanillin $/ \beta$-CD IC, (iii) vanillin $/ \gamma$-CD IC, (iv) vanillin $/ \gamma$-CD IC (coprecipitation), and (v) vanillin.

microliters of vapor was injected to the GC-MS by using a headspace injector $(\mathrm{MSH} 02-00 \mathrm{~B}$, volume $=2.5 \mathrm{~mL}$, scale $=60 \mathrm{~mm}$ ). The syringe temperature was kept the same as the incubation temperature. The separation of compounds was performed on an HP-5MS (HewlettPackard, Avondale, PA) capillary column $(30 \mathrm{~m} \times 0.25 \mathrm{~mm}$ i.d., $0.25 \mu \mathrm{m}$ film thickness). Column temperature was held at $50{ }^{\circ} \mathrm{C}$ for $2 \mathrm{~min}$ and increased to $250{ }^{\circ} \mathrm{C}$ at the rate of $20{ }^{\circ} \mathrm{C} / \mathrm{min}$ and equilibrated at this temperature for $3 \mathrm{~min}$. Helium was used as a carrier gas at a flow rate of $1.2 \mathrm{~mL} / \mathrm{min}$. Thermal desorption was carried out in the splitless mode during $2 \mathrm{~min}$. The temperatures of the ion source and the transfer line were 230 and $280^{\circ} \mathrm{C}$, respectively. The GC-MS analyses were carried out in the complete scanning mode (SCAN) in the 50-550 mass range. The vanillin peak was identified by comparing its mass spectrum with that of vanillin in the Flavor 2 and NIST 0.5 libraries. The retention time of vanillin was $8.76 \mathrm{~min}$, and the major peaks of the vanillin were 151 and 152 mass over charge.

\section{RESULTS AND DISCUSSION}

In this study, we prepared solid inclusion complexes of vanillin with three types of cyclodextrin $(\alpha-\mathrm{CD}, \beta-\mathrm{CD}$, and $\gamma$-CD) by using a freeze-drying method; in addition, a coprecipitation method was also used in the case of vanillin $/ \gamma$-CD IC. Initially, the presence of the guest molecule (vanillin) in the cyclodextrin inclusion complexes was confirmed by the FTIR studies. The characteristic absorption bands of CDs are observed at around 1030,1080, and $1155 \mathrm{~cm}^{-1}$ corresponding to the coupled $\mathrm{C}-\mathrm{C} / \mathrm{C}-\mathrm{O}$ stretching vibrations and the asymmetric stretching vibration of the $\mathrm{C}-\mathrm{O}-\mathrm{C}$ glycosidic bridge in the FTIR spectra of pure CDs (Figure 2a). The FTIR spectra of the vanillin/CD ICs are depicted in Figure 2b, and the FTIR spectrum of pure vanillin is also given for comparison. The FTIR spectrum of pure vanillin exhibited characteristic peaks at 1510,1590 , and $1665 \mathrm{~cm}^{-1}$ corresponding to stretching absorption of benzene ring and stretching of $\mathrm{C}=\mathrm{O}$ of the aldehyde group. ${ }^{21}$ The overlapping of absorption peaks of CDs and vanillin makes the identification of the individual components rather complicated in vanillin/CD ICs. Yet, the absorption bands of vanillin at 1512,1595 , and $1670 \mathrm{~cm}^{-1}$ were observed for all vanillin/CD ICs, which indicated that vanillin is present in the samples. In addition, it was noted that the characteristic absorption bands of vanillin were shifted slightly for vanillin/CD ICs when compared to pure vanillin, suggesting the host-guest interactions between $C D$ and vanillin in these samples. This is very typical for CD ICs, and FTIR peak shifts were commonly reported for various CD IC systems. ${ }^{22,23}$

The presence of vanillin in the CD ICs and their stoichiometries were determined by ${ }^{1} \mathrm{H}$ NMR studies (Figure 3 ). The peaks for vanillin observed at about $3.8,6.9,7.4$, and $9.8 \mathrm{ppm}$ correspond to different protons in vanillin. ${ }^{18,19}$ The quantity of vanillin in the CD ICs was calculated by integrating the peak ratio of the characteristic chemical shifts corresponding to vanillin and $\mathrm{CD}$. The integration of the $\mathrm{CD}$ peak at $4.8 \mathrm{ppm}^{20}$ and the vanillin peak at $9.8 \mathrm{ppm}$ was taken into account to calculate the stoichiometry of the host and guest molecules in the CD IC samples. It was found that vanillin/ $\beta$-CD IC and vanillin $/ \gamma$-CD IC have a $1: 1$ (vanillin/CD) molar ratio, whereas the ratio of vanillin/ $\alpha-\mathrm{CD}$ was $0.625: 1$, suggesting that the $1: 1$ initial molar ratio used for the formation of CD ICs was not conserved in the case of vanillin/ $\alpha$-CD IC. This suggests that $\alpha$-CD was not very effective in encapsulating vanillin, which is possibly because of the small dimension of the $\alpha$-CD cavity. Additionally, the chemical shifts for pure vanillin and the vanillin/ $\alpha$-CD system were almost the same in the NMR spectrum, indicating that vanillin and $\alpha$-CD were decomplexed in the $d_{6}$-DMSO solvent system. However, we observed differences in chemical shifts (about 0.1 ppm shift) for vanillin peaks in vanillin/ $\beta$-CD and vanillin $/ \gamma-\mathrm{CD}$ samples when compared to pure vanillin, which is due to the presence of complexation.

XRD studies were performed to investigate the crystalline structure of vanillin/CD ICs. The as-received CDs have a cagetype packing structure in which the cavity of each CD molecule is blocked by neighboring CD molecules (Figure 1c). ${ }^{24,25}$ The inclusion complexation is generally confirmed by the formation of channel-type arrangement of the $\mathrm{CD}$ molecules in which $\mathrm{CD}$ molecules are aligned and stacked on top of each other by forming cylindrical channels (Figure 1d). ${ }^{24-26}$ The XRD patterns of as-received CDs (Figure 4a) and vanillin/CD ICs (Figure 4b) have characteristic diffraction peaks in the range of $2 \theta=5-30^{\circ}$. Vanillin is a crystalline material having a distinct diffraction centered at $2 \theta \cong 13^{\circ}$ (Figure $4 \mathrm{~b}$ ). The XRD data show that the as-received $\mathrm{CDs}(\alpha-\mathrm{CD}, \beta$-CD, and $\gamma$-CD) have cage-type packing structures, which is consistent with the literature findings. ${ }^{26-28}$

The XRD patterns of vanillin/CD ICs are very different from those of as-received CDs (Figure 4b). The XRD of the vanillin/ $\alpha$-CD IC shows two broad halo diffraction patterns, indicating that the material was mostly amorphous; yet, a distinct peak 
e.

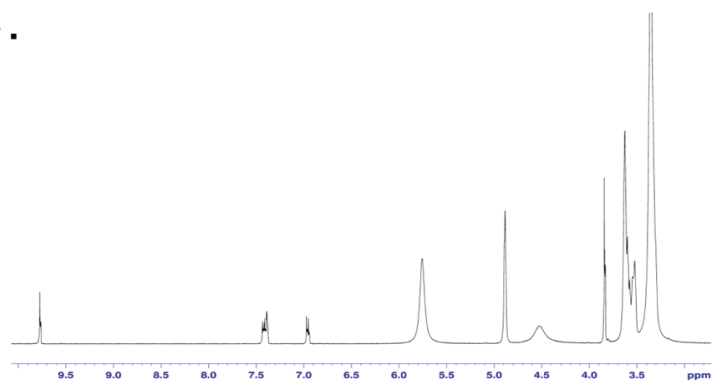

d.

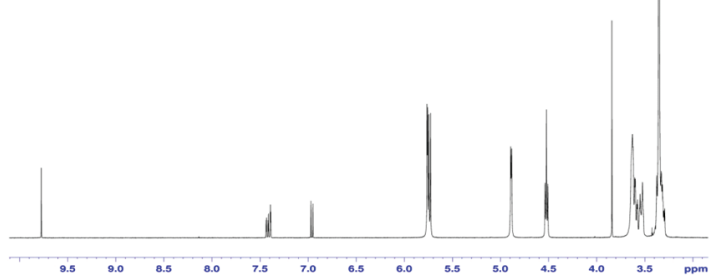

c.

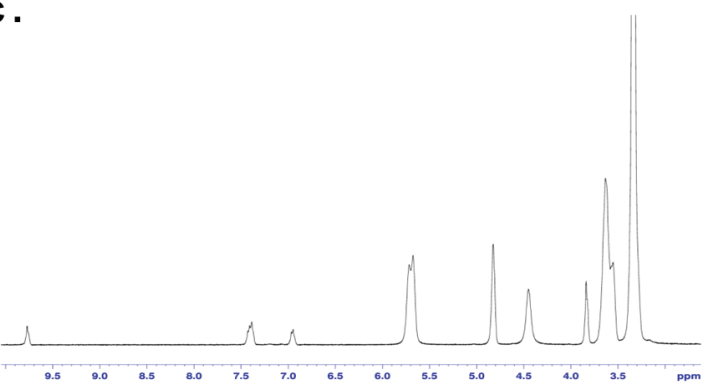

b.

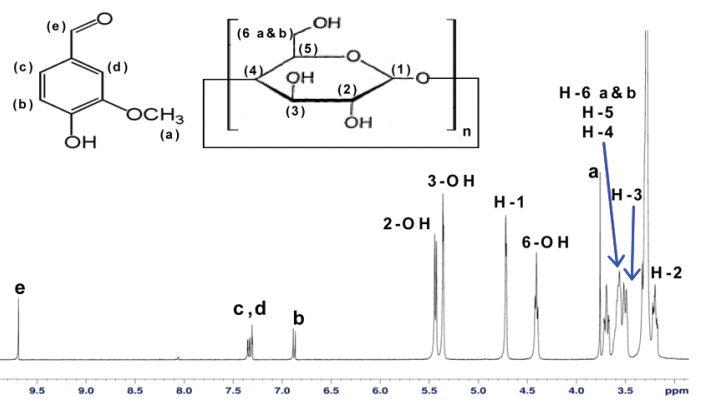

a .
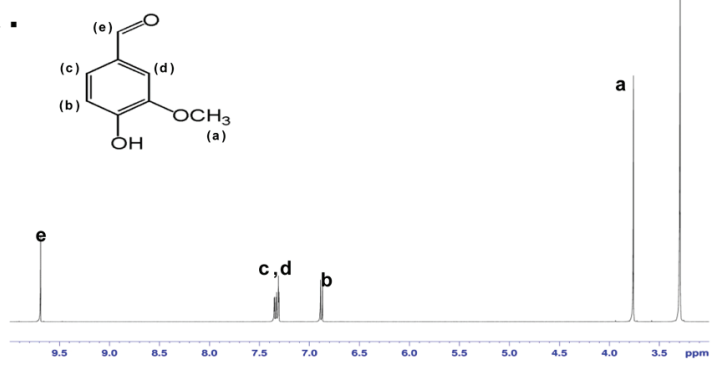

Figure 3. ${ }^{1} \mathrm{H}$ NMR spectra of (a) vanillin, (b) vanillin/ $\alpha$-CD IC, (c) vanillin/ $\beta$-CD IC, (d) vanillin $/ \gamma-$ CD IC, and (e) vanillin $/ \gamma-$ CD IC (coprecipitation).

at $2 \theta \cong 13^{\circ}$ and a weak peak at $2 \theta \cong 20^{\circ}$ were present. The $\alpha$-CD having channel-type structure has two prominent peaks centered at $2 \theta \cong 13^{\circ}$ and $2 \theta \cong 20^{\circ} .{ }^{27}$ As mentioned above, vanillin has a major diffraction peak at $2 \theta \cong 13^{\circ}$ (Figure $4 \mathrm{~b}$ ). The overlapping of vanillin and the $\alpha$-CD channel-type diffraction peaks makes the peak assignment rather complicated. The supplementary thermal analyses by DSC, which will be discussed in the following section, indicated that some free vanillin was present in the vanillin/ $\alpha$-CD IC sample. Therefore, the peak at $2 \theta \cong 13^{\circ}$ was due to both the presence of some uncomplexed free vanillin and the $\alpha-\mathrm{CD}$ channel-type packing. In short, the XRD data of vanillin $/ \alpha$-CD IC suggest that some free vanillin is present in vanillin $/ \alpha$-CD IC and that the sample was mostly amorphous, having some amount of channel-type structure.

The XRD pattern of vanillin/ $\beta$-CD IC shows two major peaks centered at $2 \theta \cong 11.8^{\circ}$ and $2 \theta \cong 17.8^{\circ}$, indicating that the $\beta$-CD adopts a channel-type packing; ${ }^{28}$ in addition, no peak at $2 \theta \cong 13^{\circ}$ for free vanillin was observed, suggesting that complete complexation of vanillin with $\beta$-CD was successful. In the case of vanillin $/ \gamma$-CD IC obtained by coprecipitation technique, the XRD pattern has a major peak at $2 \theta \cong 7.5^{\circ}$ along with minor diffractions at $2 \theta \cong 14.2^{\circ}, 15^{\circ}, 16^{\circ}, 16.8^{\circ}$, and $22^{\circ}$, confirming the tetragonal channel-type packing of $\gamma-\mathrm{CD}^{26,27}$ The XRD pattern of vanillin $/ \gamma$-CD IC obtained by freeze-drying is similar to that of the vanillin $/ \gamma$-CD IC obtained by coprecipitation method; yet, the peaks are relatively broad, indicating that the material was partially amorphous. In addition, the peak at $2 \theta \cong$ $6.2^{\circ}$ corresponding to channel structure with hexagonal packing was also observed. ${ }^{26}$ The tetragonal channel packing transforms to hexagonal packing upon removal of water residing in the interstitial sites when vacuum-dried. ${ }^{26}$ Hence, in the case of freeze-drying method, vanillin $/ \gamma$-CD IC having a mixture of tetragonal and hexagonal channel-type packing was obtained. Similar to vanillin/ $\beta$-CD IC, the vanillin peak at $2 \theta \cong 13^{\circ}$ was absent, indicating that uncomplexed vanillin was not present in both vanillin $/ \gamma$-CD IC samples. The XRD data suggested that both coprecipitation and freeze-drying methods were successful for obtaining solid vanillin $/ \gamma$-CD ICs.

The solid vanillin/CD ICs were further characterized by DSC (Figure 5a) to verify whether the vanillin was included inside the $\mathrm{CD}$ cavities or not. For instance, thermal transitions such as the melting point $\left(T_{\mathrm{m}}\right)$ for the guest molecule would be observed if there were any uncomplexed guest molecules present in the $\mathrm{CD}$ ICs. ${ }^{29-31}$ The DSC thermograms of vanillin/CD physical mixtures have shown an endothermic peak at around $77^{\circ} \mathrm{C}$, which corresponds to the melting point of vanillin (Figure $5 b$ ). The DSC thermogram of pure vanillin is also given for comparison, showing a melting point of $82{ }^{\circ} \mathrm{C}$. On the other hand, the DSC thermogram of vanillin/ $\beta$-CD IC did not show any melting peak for free vanillin, suggesting that the vanillin was fully complexed with the $\beta$-CD. Similarly, the melting peak of free vanillin was also absent in the DSC thermograms of vanillin $/ \gamma$-CD IC samples obtained by freeze-drying and coprecipitation methods, confirming that both techniques resulted in pure vanillin $/ \gamma-\mathrm{CD}$ ICs. The absence of a vanillin melting point in DSC and the absence of a diffraction peak at $2 \theta \cong 13^{\circ}$ in XRD for solid vanillin $/ \beta$-CD IC and vanillin $/ \gamma$-CD IC samples comprise strong evidence for the complete inclusion complexation of vanillin with $\beta$-CD and $\gamma$-CD. However, in the case of vanillin/ $\alpha$-CD IC, a small endothermic peak at around $75{ }^{\circ} \mathrm{C}$ was observed in the DSC thermogram due to the presence of some free vanillin in this sample. The DSC data also correlate with the XRD data as discussed in the previous section, where the XRD of vanillin $/ \alpha$-CD IC has a diffraction peak for some uncomplexed vanillin crystals. 

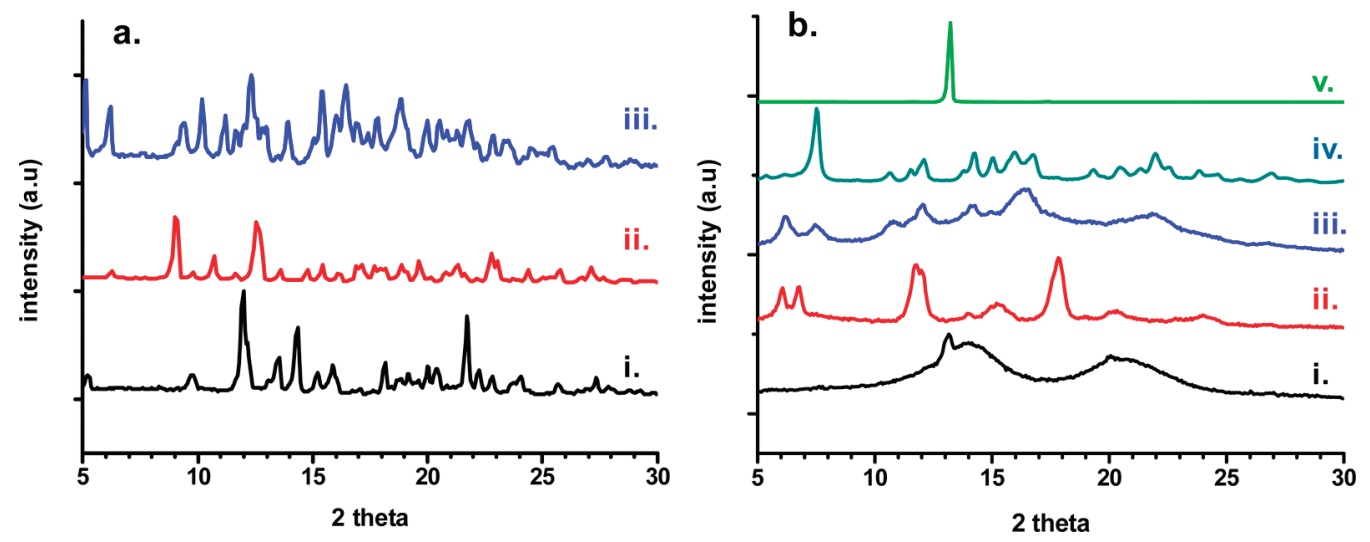

Figure 4. XRD patterns of (a) as-received (i) $\alpha$-CD, (ii) $\beta$-CD, and (iii) $\gamma$-CD and (b) (i) vanillin $/ \alpha$-CD IC, (ii) vanillin/ $\beta$-CD IC, (iii) vanillin $/ \gamma$-CD IC, (iv) vanillin $/ \gamma$-CD IC (coprecipitation), and (v) vanillin.
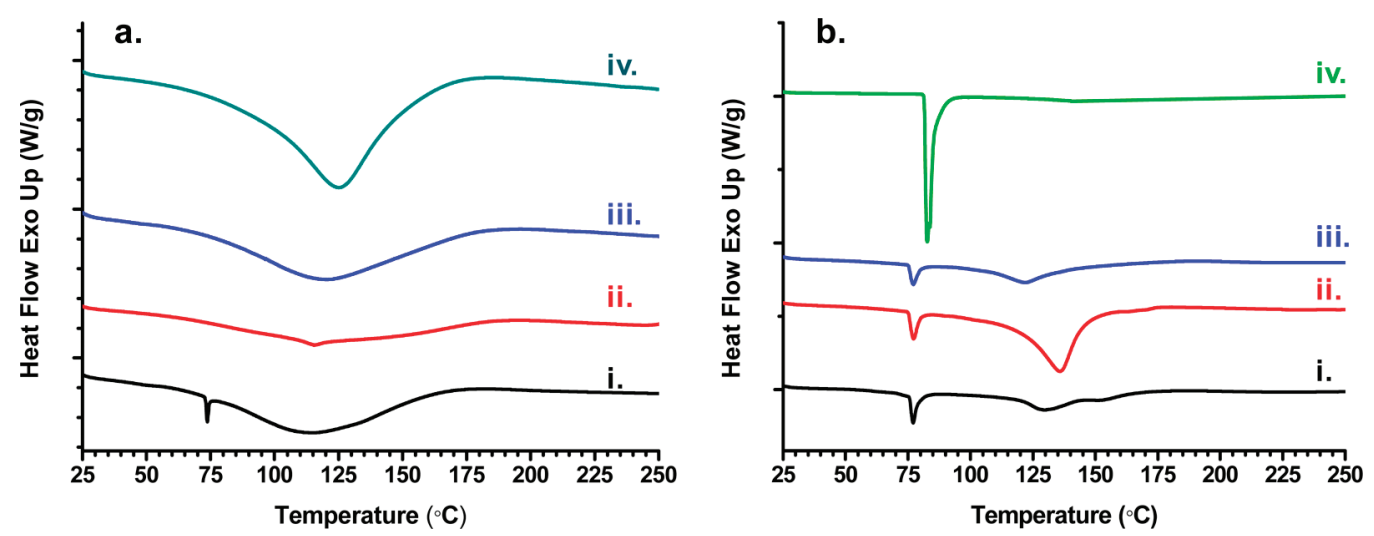

Figure 5. DSC thermograms of (a) (i) vanillin/ $\alpha$-CD IC, (ii) vanillin/ $\beta$-CD IC, (iii) vanillin $/ \gamma$-CD IC, and (iv) vanillin $/ \gamma$-CD IC (coprecipitation) and (b) (i) vanillin $/ \alpha$-CD physical mixture, (ii) vanillin $/ \beta$-CD physical mixture, (iii) vanillin $/ \gamma$-CD physical mixture, and (iv) vanillin.

For CD ICs, the thermal stability and the evaporation of the volatile guest molecules shift to higher temperature upon inclusion complexation due to guest-host interactions. ${ }^{8,26}$ Hence, TGA was carried out for vanillin/CD ICs to determine the thermal stability and evaporation of the vanillin included inside the CD cavities (Figure 6). The TGAs of vanillin/CD physical mixtures and pure vanillin were also performed for comparison (Figure 6). The TGA thermogram of pure vanillin has a major weight loss observed in the range of $80-200{ }^{\circ} \mathrm{C}$, showing that vanillin has a volatile nature. TGA thermograms of vanillin/CD ICs showed three weight losses, the initial weight loss below $100{ }^{\circ} \mathrm{C}$ is due to water loss, the second weight loss between 150 and $300{ }^{\circ} \mathrm{C}$ is due to the evaporation/degradation of vanillin, and the major weight loss above $300{ }^{\circ} \mathrm{C}$ corresponds to the main thermal degradation of CD. The TGA thermograms of vanillin/ CD ICs showed that thermal degradation of vanillin occurred over a much higher temperature range $\left(150-300{ }^{\circ} \mathrm{C}\right)$ when compared to pure vanillin $\left(80-200{ }^{\circ} \mathrm{C}\right)$, confirming that the thermal stability of vanillin increased due to inclusion complexation with CDs. For vanillin/CD physical mixtures, three weight losses were observed: the one below $100{ }^{\circ} \mathrm{C}$ is attributed to removal of water, the second one in the range of $80-200{ }^{\circ} \mathrm{C}$ corresponds to the evaporation of vanillin, and the one above $300{ }^{\circ} \mathrm{C}$ is due to the main thermal degradation of $\mathrm{CD}$. The temperature range for evaporation of vanillin for vanillin/CD physical mixtures is similar to that of pure vanillin. However, in the case of vanillin/CD ICs, the thermal stability of vanillin shifted to a much higher temperature owing to the strong interactions between vanillin and the CD cavity in the CD ICs.

The amount of vanillin was calculated for solid vanillin/CD IC samples from the TGA data. For vanillin/ $\beta$-CD IC, the amount of vanillin was $\sim 11 \%(\mathrm{w} / \mathrm{w}$, with respect to $\beta$-CD), which correlates with the initial amount of vanillin used for the complexation. This corresponds to a 1:1 molar ratio complexation between the $\beta$-CD and vanillin, correlating with the NMR data; the initial amount of vanillin was preserved, and no loss of guest molecule occurred during the formation and storage of vanillin/ $\beta$-CD IC. The amount of vanillin was calculated as $\sim 10 \%(\mathrm{w} / \mathrm{w}$, with respect to $\gamma$-CD) for both vanillin $/ \gamma$-CD IC samples obtained by freeze-drying and coprecipitation method. Similar to vanillin/ $\beta$-CD IC, vanillin/ $\gamma$-CD IC samples have also a $1: 1$ molar ratio complexation between the $\gamma$-CD and vanillin as observed in the NMR study, and the initial amount of vanillin was preserved during the formation and storage of these samples. In the case of vanillin/ $\alpha$-CD IC, the amount of vanillin was calculated as $\sim 8 \%$ (w/w, with respect to $C D$ ), which is lower than the initial weight percent $(\sim 13 \%, w / w)$. As DSC and XRD data confirmed, some uncomplexed vanillin was present in the sample, and it is likely that free vanillin could not be preserved during the storage of the vanillin/ $\alpha$-CD IC sample.

The temperature stability and release characteristics of vanillin/CD ICs were also studied by headspace GC-MS. 

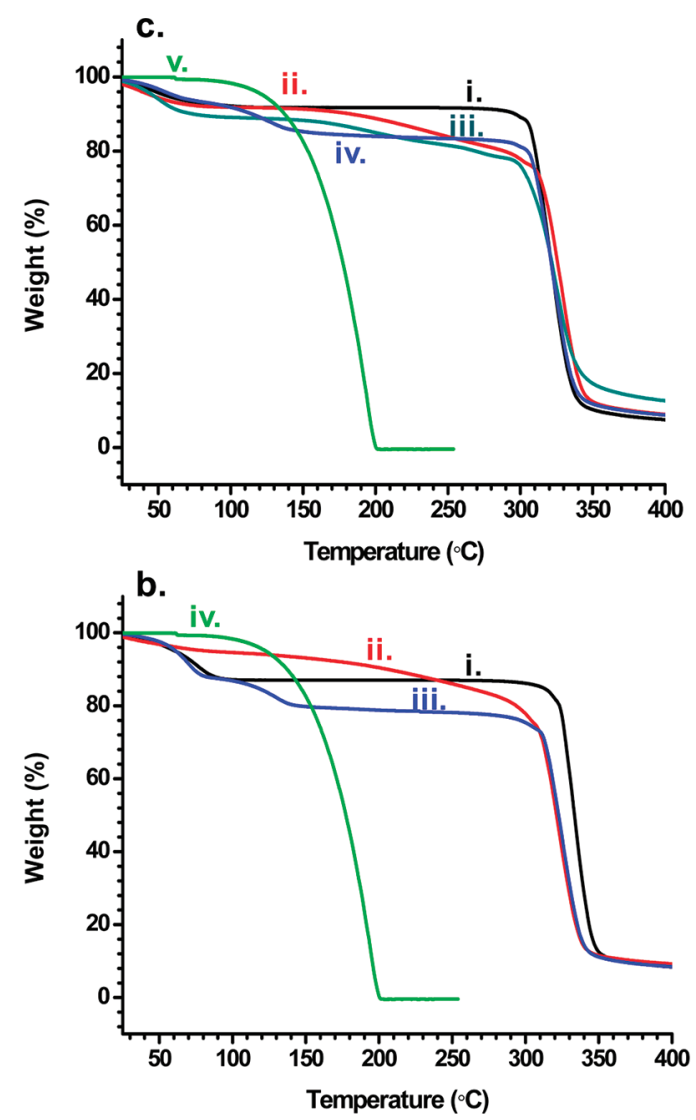

a.

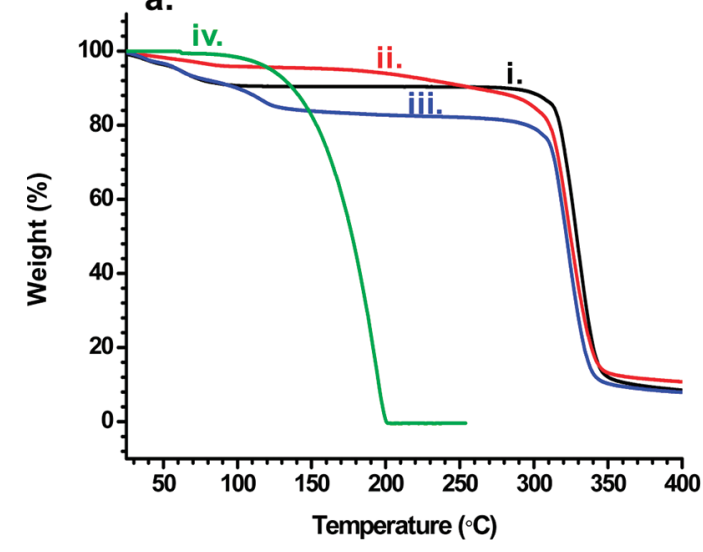

Figure 6. TGA thermograms of (a) (i) $\alpha$-CD, (ii) vanillin $/ \alpha$-CD IC, (iii) vanillin $/ \alpha$-CD physical mixture, and (iv) vanillin; (b) (i) $\beta-\mathrm{CD}$, (ii) vanillin $/ \beta$-CD IC, (iii) vanillin/ $\beta$-CD physical mixture, and (iv) vanillin; and (c) (i) $\gamma$-CD, (ii) vanillin $/ \gamma$-CD IC, (iii) vanillin $/ \gamma$-CD IC (coprecipitation), (iv) vanillin $/ \gamma$-CD physical mixture, and (v) vanillin.

The vanillin/CD physical mixtures were also analyzed for comparison. The release of the vanillin from each sample was quantified from the corrected peak area calculated at $8.76 \mathrm{~min}$ from each chromatogram. The relative release of vanillin at different incubation temperatures $\left(35,50,75\right.$, and $\left.100^{\circ} \mathrm{C}\right)$ was calculated by dividing the corrected peak areas obtained at different temperatures with the one obtained at $35{ }^{\circ} \mathrm{C}$ for each sample. The relative release of the vanillin with increasing temperature is summarized in Table 1. It is evident that the release of vanillin was much slower for vanillin/CD ICs at all temperatures when compared to the vanillin/CD physical mixtures. In addition, it
Table 1. Corrected Peak Areas ${ }^{a}$ Calculated at $8.76 \mathrm{~min}$ from Headspace GC-MS Chromatograms for Vanillin/CD ICs and Vanillin/CD Physical Mixtures Agitated at Different Incubation Temperatures

\begin{tabular}{lcccc}
\multicolumn{1}{c}{ sample } & $35^{\circ} \mathrm{C}$ & $50{ }^{\circ} \mathrm{C}$ & $75{ }^{\circ} \mathrm{C}$ & $100{ }^{\circ} \mathrm{C}$ \\
vanillin $/ \alpha$-CD IC & $\times 1$ & $\times 52$ & $\times 736$ & $\times 3002$ \\
vanillin $/ \alpha$-CD physical mixture & $\times 1$ & $\times 75$ & $\times 981$ & $\times 4898$ \\
& & & & \\
vanillin $/ \beta$-CD IC & $\times 1$ & $\times 11$ & $\times 108$ & $\times 1119$ \\
vanillin $/ \beta$-CD physical mixture & $\times 1$ & $\times 41$ & $\times 519$ & $\times 2083$ \\
& & & & \\
vanillin $/ \gamma$-CD IC & $\times 1$ & $\times 10$ & $\times 51$ & $\times 912$ \\
vanillin $/ \gamma$-CD IC (coprecipitation) & $\times 1$ & $\times 10$ & $\times 63$ & $\times 283$ \\
vanillin $/ \gamma$-CD physical mixture & $\times 1$ & $\times 45$ & $\times 385$ & $\times 1486$ \\
${ }^{a}$ All of the peak area values were divided by the peak area obtained at \\
$35^{\circ} \mathrm{C}$ for each sample.
\end{tabular}

was observed that as the temperature was increased, the relative amount of vanillin released was in the order vanillin $/ \alpha-\mathrm{CD}>$ vanillin $/ \beta$-CD $>$ vanillin $/ \gamma-\mathrm{CD}$ for $\mathrm{CD}$ IC samples, indicating that the strength of interaction between vanillin and the $\mathrm{CD}$ cavity was in the order $\gamma$-CD $>\beta$-CD $>\alpha$-CD. Moreover, in the case of vanillin $/ \gamma$-CD IC, it was noted that the coprecipitation method was more effective for the stabilization and sustained release of vanillin at high temperature $\left(100^{\circ} \mathrm{C}\right)$ when compared to the freeze-drying method. In the coprecipitation method, the vanillin $/ \gamma$-CD IC sample was washed several times to remove uncomplexed and/or loosely complexed vanillin; however, the vanillin $/ \gamma$-CD IC sample obtained by the freeze-drying method may contain some amount of loosely complexed vanillin, and this may be why CD ICs prepared by the coprecipitation method were more effective for the stabilization of vanillin at high temperature. In short, the headspace GC-MS studies showed that the temperature release profile of vanillin was different for each CD IC sample and highly dependent on the type of CD. The reason for the formation of a more stable inclusion complex between vanillin and $\gamma$-CD is possibly because of the bigger cavity size of $\gamma-\mathrm{CD}$, resulting in a better fit and size match between the vanillin molecule and the $\gamma$-CD cavity.

In conclusion, the formation of solid CD ICs was successfully achieved between vanillin and three types of $\mathrm{CDs}(\alpha-\mathrm{CD}, \beta-\mathrm{CD}$, and $\gamma$-CD). The inclusion complexation of vanillin with $\beta$-CD and $\gamma$-CD having a 1:1 molar ratio was quite successful, whereas $\alpha$-CD has shown poor complexation ability. Vanillin has a volatile nature; however, higher thermal stability and sustained release of vanillin at high temperatures were attained by forming vanillin/CD ICs. The thermal evaporation/degradation of vanillin shifted to a much higher temperature range $\left(150-300^{\circ} \mathrm{C}\right)$ for vanillin/CD IC samples when compared to pure vanillin $\left(80-200{ }^{\circ} \mathrm{C}\right)$ or vanillin/CD physical mixtures. Additionally, headspace GC-MS analyses showed that the relative amount of vanillin released as a function of temperature was lowest for vanillin $/ \gamma$-CD IC and highest for vanillin $/ \alpha$-CD IC, suggesting that the strength of interaction between vanillin and the $\mathrm{CD}$ cavity was in the order $\gamma-\mathrm{CD}>\beta$-CD $>\alpha$-CD. In brief, $\mathrm{CD}$ ICs of a widely used flavor/fragrance, vanillin, were achieved with improved thermal stability, and such solid vanillin/CD ICs can be quite applicable in the food industry, active food packaging, the textile industry, etc. 


\section{AUTHOR INFORMATION}

\section{Corresponding Author}

*E-mail: tamer@unam.bilkent.edu.tr. Phone: (+90)3122903571. Fax: (+90)3122664365.

\section{Funding Sources}

The State Planning Organization (DPT) of Turkey is acknowledged for the support of UNAM-Institute of Materials Science and Nanotechnology. T.U. acknowledges EU FP7-PEOPLE-2009-RG Marie Curie-IRG for funding the NANOWEB (PIRG06-GA-2009256428 ) project. F.K. acknowledges TUBITAK-BIDEB for the national graduate study scholarship.

\section{REFERENCES}

(1) Szejtli, J. Introduction and general overview of cyclodextrin chemistry. Chem. Rev. 1998, 98, 1743-1754.

(2) Del Valle, E. M. M. Cyclodextrins and their uses: a review. Process Biochem. 2004, 39 (9), 1033-1046.

(3) Hedges, A. Industrial applications of cyclodextrins. Chem. Rev. 1998, 98, 2035-2044.

(4) Singh, M.; Sharma, R.; Banerjee, U. Biotechnological applications of cyclodextrins. Biotechnol. Adv. 2002, 20 (5-6), 341-359.

(5) Szejtli, J. Cyclodextrins in the textile industry. Starch/Staerke 2003, 55 (5), 191-196.

(6) Szente, L.; Szejtli, J. Cyclodextrins as food ingredients. Trends Food Sci. Technol. 2004, 15 (3-4), 137-142.

(7) Buschmann, H.; Knittel, D.; Schollmeyer, E. New textile applications of cyclodextrins. J. Incl. Phenom. Macrocycl. Chem. 2001, 40 (3), 169-172.

(8) Marcolino, V. A.; Zanin, G. M.; Durrant, L. R.; Benassi, M. D. T.; Matioli, G. Interaction of curcumin and bixin with $\beta$-cyclodextrin: complexation methods, stability, and applications in food. J. Agric. Food Chem. 2011, 59, 3348-3357.

(9) Kant, A.; Linforth, R. S. T.; Hort, J.; Taylor, A. J. Effect of $\beta$-cyclodextrin on aroma release and flavor perception. J. Agric. Food Chem. 2004, 52, 2028-2035.

(10) Rekharsky, M. V.; Inoue, Y. Complexation thermodynamics of cyclodextrins. Chem. Rev. 1998, 98, 1875-1918.

(11) Connors, K. A. The stability of cyclodextrin complexes in solution. Chem. Rev. 1997, 97, 1325-1358.

(12) Reineccius, T. A.; Reineccius, G. A.; Peppard, T. L. The effect of solvent interactions on $\alpha$-, $\beta$-, and $\gamma$-cyclodextrin/flavor molecular inclusion complexes. J. Agric. Food Chem. 2005, 53, 388-392.

(13) Anklam, E.; Gaglione, S.; Müller, A. Oxidation behaviour of vanillin in dairy products. Food Chem. 1997, 60, 43-51.

(14) Karathanos, V.; Mourtzinos, I.; Yannakopoulou, K; Andrikopoulos, N. Study of the solubility, antioxidant activity and structure of inclusion complex of vanillin with $\beta$-cyclodextrin. Food Chem. 2007, 101 (2), 652-658.

(15) Tai, A.; Sawano, T.; Yazama, F.; Ito, H. Evaluation of antioxidant activity of vanillin by using multiple antioxidant assays. Biochim. Biophys. Acta-Gen. Subj. 2011, 1810, 170-177.

(16) Lo Nostro, P.; Fratoni, L.; Baglioni, P. Modification of a cellulosic fabric with $\beta$-cyclodextrin for textile finishing applications. J. Incl. Phenom. 2002, 44, 423-427.

(17) Astray, G.; Mejuto, J. C.; Morales, J.; Rial-Otero, R.; SimalGándara, J. Factors controlling flavors binding constants to cyclodextrins and their applications in foods. Food Res. Int. 2010, 43, 1212-1218.

(18) Divakar, S. Structure of a $\beta$-cyclodextrin-vanillin inclusion complex. J. Agric. Food Chem. 1990, 38, 940-944.

(19) Pirnau, A.; Bogdan, M.; Floare, C. G. NMR spectroscopic characterization of $\beta$-cyclodextrin inclusion complex with vanillin. J. Phys. Conf. Ser. 2009, 182, 012013.

(20) Divakar, S.; Maheswaran, M. Structural studies on inclusion compounds of $\beta$-cyclodextrin with some substituted phenols. J. Incl. Phenom. Macrocycl. Chem. 1997, 27, 113-126.
(21) Peng, H.; Xiong, H.; Li, J.; Xie, M.; Liu, Y.; Bai, C.; Chen, L. Vanillin cross-linked chitosan microspheres for controlled release of resveratrol. Food Chem. 2010, 121, 23-28.

(22) Singh, R.; Bharti, N.; Madan, J.; Hiremath, S. Characterization of cyclodextrin inclusion complexes - a review. J. Pharm. Sci. Technol. 2010, 2, 171-183.

(23) Lin, S. Y.; Hsu, C. H.; Sheu, M. T. Curve-fitting FTIR studies of loratadine/hydroxypropyl- $\beta$-cyclodextrin inclusion complex induced by co-grinding process. J. Pharm. Biomed. Anal. 2010, 53, 799-803.

(24) Saenger, W.; Jacob, J.; Gessler, K.; Steiner, T.; Hoffmann, D.; Sanbe, H.; Koizumi, K.; Smith, S.; Takaha, T. Structures of the common cyclodextrins and their larger analogues beyond the doughnut. Chem. Rev. 1998, 98, 1787-1802.

(25) Harata, K. Structural aspects of stereo differentiation in the solid state. Chem. Rev. 1998, 98, 1803-1828.

(26) Uyar, T.; Hunt, M.; Gracz, H.; Tonelli, A. Crystalline cyclodextrin inclusion compounds formed with aromatic guests: guest-dependent stoichiometries and hydration-sensitive crystal structures. Cryst. Growth Des. 2006, 6, 1113-1119.

(27) Rusa, C.; Bullions, T.; Fox, J.; Porbeni, F.; Wang, X.; Tonelli, A. Inclusion compound formation with a new columnar cyclodextrin host. Langmuir 2002, 18, 10016-10023.

(28) Harada, A.; Okada, M.; Li, J.; Kamachi, M. Preparation and characterization of inclusion complexes of poly(propylene glycol) with cyclodextrins. Macromolecules 1995, 28 (24), 8406-8411.

(29) Giordano, F.; Novak, C.; Moyano, J. R. Thermal analysis of cyclodextrins and their inclusion compounds. Thermochim. Acta 2001, 380, 123-151.

(30) Uyar, T.; Shafei, A.; Wang, X.; Hacaloglu, J.; Tonelli, A. E. The solid channel structure inclusion complex formed between guest styrene and host $\gamma$-cyclodextrin. J. Incl. Phenom. Macrocycl. Chem. 2006, 55, 109-121.

(31) Celebioglu, A.; Uyar, T. Electrospinning of polymer-free nanofibers from cyclodextrin inclusion complexes. Langmuir 2011, 27, 6218-6226. 